\title{
Efectos diferenciales de la atención y la claridad emocional sobre la percepción de estresores académicos y las respuestas de estrés de estudiantes de fisioterapia
}

\author{
Differential effects of attention and emotional clarity on the perception of \\ academic stressors and stress responses of physiotherapy students
}

\author{
R.G. Cabanach ${ }^{\mathrm{a}}$, A. Souto-Gestal ${ }^{\mathrm{b}}$, L. González Doniz ${ }^{\mathrm{b}}$, S. Souto Camba ${ }^{\mathrm{b}}$ \\ ${ }^{a}$ Grupo de investigación en Intervención Psicosocial y Rehabilitación Funcional, Departamento de \\ Psicología Evolutiva y de la Educación, Red Salud, Bienestar físico y psicológico, Universidad de A \\ Coruña, A Coruña, España \\ ${ }^{b}$ Grupo de investigación en Intervención Psicosocial y Rehabilitación Funcional, Departamento de \\ Fisioterapia, Red Salud, Bienestar físico y psicológico, Universidad de A Coruña, A Coruña, España
}

\begin{abstract}
Resumen
Objetivo. Analizar los efectos diferenciales de los niveles de atención y claridad emocional sobre la percepción de estresores académicos y las respuestas de estrés de estudiantes de fisioterapia.

Material y método. Se evaluaron los niveles de atención y claridad emocional con la Escala de Dificultades en la Regulación Emocional y la percepción de estresores académicos y respuestas de estrés con el Cuestionario de Estrés Académico de 500 estudiantes de fisioterapia de diferentes universidades españolas. Los estudiantes fueron clasificados en función de su grado de atención y claridad emocional (alto, medio y bajo), y se compararon la percepción de estresores y respuestas de estrés mediante un análisis de la varianza. Resultados. Los estudiantes con elevada claridad emocional valoran el entorno académico como menos amenazante y muestran menores respuestas psicofisiológicas de estrés en comparación con los estudiantes con niveles medios o bajos de claridad emocional. Por el contrario, los estudiantes con baja atención emocional únicamente valoran como más estresantes aquellas situaciones relacionadas con las creencias sobre el rendimiento y sobre el valor de los contenidos, en comparación con los estudiantes con alta atención emocional.

Conclusiones. Mientras la claridad emocional presenta una función moderadora sobre la valoración del entorno académico en términos de amenaza y sobre los efectos físicos y psicológicos del estrés, el papel de la atención emocional resulta menos relevante y únicamente se relaciona con la percepción de estresores vinculados a las creencias del estudiante.
\end{abstract}

\begin{abstract}
Objective. To analyze the differential effects of levels of emotional attention and emotional clarity on the perception of academic stressors and stress responses of physiotherapy students.

Material and method. Levels of emotional attention and emotional clarity were measured with Difficulties in Emotional Regulation Scale, and perception of academic stressors and stress responses were evaluated with Academic Stress Questionnaire in 500 physiotherapy students from different Spanish universities. Students were classified according to their degree of emotional attention and emotional clarity in high, medium and low, and the perception of stressors and stress responses were compared through analysis of variance.

Results. Students with high emotional clarity assess the academic environment as less threatening and show lower psychophysiological stress responses in comparison to students with medium or low levels of emotional clarity. Conversely, students with low emotional attention only assess more stressful situations related to beliefs about the performance and the value of content than students with high emotional attention.
\end{abstract}


Conclusions. While emotional clarity has a moderating role on the valuation of the academic environment as threatening and on the physical and psychological effects of stress, the role of emotional attention is less relevant and only relates with the perception of stressors linked to the beliefs of the student.

\section{Palabras clave}

Estrés fisiológico; Inteligencia emocional; Fisioterapia; Atención

Keywords

Physiological stress; Emotional intelligence; Physical therapy specialty; Attention

\section{Introducción}

El estrés parece afectar a un porcentaje significativo de estudiantes universitarios, como consecuencia de diversas razones: sobrecarga en sus tareas, problemas en la gestión del tiempo, elevados niveles de ansiedad cuando han de intervenir en clase, la realización de exámenes, etc. En concreto, existen evidencias que muestran que los estudiantes de titulaciones sanitarias presentan con frecuencia altos niveles de estrés en comparación con otras titulaciones ${ }^{1}$.

En la investigación del estrés entre estudiantes universitarios, la mayoría de los trabajos hasta la fecha se han centrado en identificar un conjunto de estímulos o condiciones potencialmente estresantes y a asociarlos a una serie de resultados de estrés ${ }^{2}$. En los últimos años han surgido nuevas líneas que tratan de analizar el papel que desempeñan diferentes características psicológicas o recursos intrapsíquicos como moderadores del estrés en estudiantes ${ }^{3-5}$.

Entre los diferentes recursos intrapsíquicos considerados, el análisis de las competencias relacionadas con el manejo y regulación de las emociones como importante factor protector del estrés en estudiantes sanitarios ha sido objeto de un incipiente interés ${ }^{6-8}$. Se podrían sintetizar los resultados de estos trabajos afirmando que la regulación emocional constituye un factor relevante en la prevención del estrés y la promoción del ajuste y el bienestar académico de los estudiantes de estas titulaciones.

Dentro de la regulación emocional, Gratz y Roemer ${ }^{9}$ diferencian varias dimensiones: la atención y conciencia de las emociones, la claridad o comprensión de las emociones, la aceptación de las emociones, la capacidad para controlar las conductas impulsivas y comportarse de acuerdo con los objetivos deseados cuando se experimentan emociones negativas, y la capacidad para utilizar estrategias de regulación emocional contextualmente apropiadas que permitan modular las respuestas emocionales con el objetivo de satisfacer los objetivos individuales y las demandas situacionales.

Las 2 primeras dimensiones se incluirían dentro del componente experiencial de las emociones, que posibilitarían el procesamiento básico de la información emocional. Un uso eficaz de esta información emocional incidiría positivamente en su uso estratégico y, en consecuencia, en el manejo y gestión de las emociones.

La primera de ellas, la atención emocional, posibilita el conocimiento y la conciencia de las propias emociones, lo que representa el primer paso para su posterior comprensión, control y regulación. Stratton et al. ${ }^{10}$ encuentran una correlación positiva entre atención a las emociones informada por estudiantes de medicina y sus habilidades de comunicación y exploración física de pacientes. Posteriormente, en un estudio longitudinal ${ }^{11}$ comprobaron que, tras 2 años de formación clínica, los estudiantes de medicina mostraron una reducción significativa en las puntuaciones de atención a las emociones, así como niveles superiores de estrés percibido. Sin embargo, otros 
trabajos con estudiantes universitarios, como el de Extremera et al. ${ }^{12}$, informan que mayores niveles de atención emocional se relacionan con mayores niveles de estrés percibido. Ello podría explicarse por las dificultades que muestran los individuos que se caracterizan por supervisar en todo momento la evolución de su estado de ánimo y realizan un enorme esfuerzo por tratar de comprenderlo. Este proceso resulta especialmente desadaptativo cuando este alto nivel de atención no va acompañado de la discriminación de las causas, razones y consecuencias (esto es, de claridad emocional). Una alta conciencia emocional acompañada por una baja claridad emocional promoverían la creencia de falta de capacidad para modificar los estados emocionales, con el consiguiente coste para la salud mental y la realización personal ${ }^{13}$. Estas personas corren el peligro de verse envueltas en una espiral emocional o círculo vicioso que, en lugar de aliviar su estado de ánimo, los conduce a un proceso pseudorreflexivo fuera de su control, prolongando así un estado de ánimo negativo a través de estrategias rumiativas, evitativas o impulsivas ${ }^{14,15}$.

En consecuencia, si bien existe un elevado grado de acuerdo en que el manejo eficaz de las emociones desempeña una función protectora ante el estrés académico, no está clara la relación que el componente experiencial de las mismas (la atención y la claridad emocional) mantiene con el estrés, Ni si, dentro de este componente, la atención emocional y la claridad emocional tienen la misma relación con el estrés académico, lo que no parece concordar con los antecedentes teóricos. El objetivo del presente trabajo es contribuir a clarificar cuál es la relación de estas dimensiones con el estrés académico de los estudiantes de la titulación de fisioterapia -en concreto, con la percepción de estresores académicos y con las manifestaciones o efectos-. De acuerdo con este objetivo, las hipótesis establecidas serían 2: 1) Los estudiantes de fisioterapia que informen de mayores niveles de atención emocional percibirán en mayor medida que las situaciones académicas constituyen una realidad estresante. Asimismo, experimentarán una mayor frecuencia de respuestas psicofisiológicas de estrés como irascibilidad, alteraciones del sueño, agotamiento físico o pensamientos negativos. 2) Por el contrario, los estudiantes de fisioterapia que informen de mayores niveles de claridad emocional percibirán en menor medida que las situaciones académicas constituyen una realidad estresante. Asimismo, experimentarán una menor frecuencia de respuestas psicofisiológicas de estrés como irascibilidad, alteraciones del sueño, agotamiento físico o pensamientos negativos.

\section{Material y métodos}

\section{Participantes}

La muestra utilizada estaba integrada por 500 estudiantes de la titulación de Fisioterapia de diferentes universidades españolas (A Coruña, Europea de Madrid, Granada, Rey Juan Carlos y Vigo). La edad de los estudiantes oscilaba entre 18 y 39 años, siendo la edad media de 21,06años y la desviación típica de 3,74. Del total de la muestra, el 74\% eran mujeres y el 26\% eran varones, lo que supone una representación bastante fiel de la habitual distribución por sexo en esta titulación, donde existe una clara mayoría femenina ${ }^{16,17}$.

Los sujetos fueron seleccionados a partir de un muestreo probabilístico por conglomerados de una etapa, con el fin de respetar el agrupamiento natural de los estudiantes. Por ello se consideró como conglomerado cada grupo clase integrado por aquellos estudiantes que voluntariamente colaboraron en la cumplimentación de los cuestionarios (muestra aceptante). 


\section{Instrumentos de medida}

Las variables del estudio y los instrumentos utilizados fueron:

\section{Atención y claridad emocional:}

Se empleó la Escala de Dificultades en la Regulación Emocional, adaptación española ${ }^{18}$ de la Difficulties in Emotion Regulation Scale (DERS) ${ }^{9}$.

De los 5 factores identificados tanto en la escala original como en la adaptación española, nosotros empleamos en este trabajo 2 de ellos. Se trata de las dimensiones de «desatención»y de «confusión emocional», que, siguiendo la justificación realizada en el trabajo de Cabanach et al. ${ }^{19}$, se han denominado como atención emocional y claridad emocional, respectivamente.

La dimensión de atención emocional está integrada por 5 ítems que valoran el grado de atención o conciencia que el individuo tiene de sus estados emocionales (p. ej..., «Presto atención a cómo me siento»). La de claridad emocional recoge 4 ítems que informan acerca del grado de comprensión que el sujeto tiene de sus emociones (p. ej.,., «Tengo dificultades para comprender mis sentimientos»). Las respuestas se realizan en una escala Likert de 5 puntos, desde «nunca» (1) hasta «siempre» (5). El coeficiente $\alpha$ de Cronbach para el total de la escala fue de $\alpha=0,93$, mientras que arrojó valores de 0,77 y 0,74 para las dimensiones de atención y claridad emocional, respectivamente.

\section{Percepción de estresores académicos:}

Para la medición de los estresores académicos, hemos utilizado la escala de estresores $(\text { ECEA })^{20}$, que forma parte del Cuestionario de Estrés Académico. Está integrada por un total de 54 ítems que miden el grado en que el estudiante percibe situaciones o circunstancias del contexto académico como un peligro o una amenaza. Las respuestas a cada uno de los ítems se realizan sobre una escala tipo Likert de 5 puntos, en la que el estudiante indica la frecuencia con la que experimenta determinados estímulos estresantes, desde «nunca» (1) hasta «siempre» (5). Los 54 ítems se agrupan en un total de 8 factores que explican un $66,97 \%$ de la varianza total, Este modelo factorial obtenido replica de forma casi idéntica el original. Para la realización del análisis factorial exploratorio se ha empleado como método de extracción el análisis de componentes principales y como método de rotación la normalización Varimax con Kaiser. El valor de la medida de adecuación de Kaiser-Meyer-Olkin (KMO) es de 0,952, lo que, unido a un nivel de significación de la prueba de esfericidad de Bartlett igual a 0,000, indica que es correcto proceder a la factorización de las variables. Los 8 factores son los siguientes: deficiencias metodológicas del profesorado (p. ej..., «Me pongo nervioso o me inquieto cuando no tengo claro qué exigen en las distintas materías»), sobrecarga del estudiante (p. ej..., «Me pongo nervioso o me inquieto por el excesivo tiempo que debo dedicarle a la realización de las actividades académicas»), creencias sobre el rendimiento académico (p. ej..,. «Me pongo nervioso o me inquieto porque desconozco si mi progreso académico es adecuado»), intervenciones en público (p. ej..., «Me pongo nervioso o me inquieto al hacer una exposición o al hablar en público durante un cierto tiempo»), clima social negativo (p. ej.,., «Me pongo nervioso o me siento inquieto por la ausencia de un buen ambiente en clase»), carencia de valor de los contenidos (p. ej..., «Me preocupa que lo que estoy estudiando tiene una escasa utilidad futura»), exámenes (p. ej.,., «Me pongo nervioso o me inquieto cuando tengo exámenes») y dificultades de participación (p. ej..., «Me pongo nervioso o me siento inquieto por- que no está en mi mano plantear los trabajos, tareas o actividades como me gustaría»). Los valores de consistencia interna de esta escala se mostraron excelentes, con una de Cronbach para el total de la escala de 0,97 , oscilando los 8 factores identificados entre 0,83 y 0,94 . 


\section{Respuestas psicofisiológicas de estrés:}

La respuesta de estrés fue medida con la escala de respuesta (RCEA) ${ }^{21}$, que forma parte del Cuestionario de Estrés Académico. Se compone de 22 ítems que miden síntomas vinculados a los componentes cognitivos, conductuales, afectivos y somáticos relacionados con el estrés académico. Las respuestas a cada uno de los ítems se realizan sobre una escala tipo Likert de 5 puntos, en la que el estudiante indica la frecuencia con la que experimenta determinados síntomas, desde «nunca» (1) hasta «siempre» (5). Los datos fueron sometidos a un análisis factorial exploratorio, empleando como método de extracción el análisis de componentes principales y como método de rotación la normalización Varimax con Kaiser. El valor de la medida de adecuación muestral de KMO fue de 0,940, lo que, junto con un nivel de significación de la prueba de esfericidad de Bartlett igual a 0,000, indica que resulta oportuno llevar a cabo la factorización de las variables. Estos 22 ítems se agrupan en 4 factores que explican el 72,67\%, y se denominan: agotamiento físico (p. ej..., «En las últimas semanas me siento fatigado/a cuando me levanto por la mañana y tengo que enfrentarme a otra jornada de trabajo»), dificultades con el sueño (p. ej..,., «En las últimas semanas me cuesta quedarme dormido/a»), irascibilidad (p. ej..., «En las últimas semanas estoy irritable») y pensamientos negativos (p. ej.,., «En las últimas semanas tiendo a resaltar mis fracasos y a desvalorizar mis éxitos»). El valor a de Conbrach fue de 0,93 para el total de la escala, y osciló entre 0,86 y 0,93 para cada uno de los 4 factores anteriormente descritos.

\section{Procedimiento}

El estudio se llevó a cabo siguiendo un diseño ex post facto prospectivo simple de corte transversal. Se procedió a la recogida de los datos relativos a las variables objeto de análisis en cada uno de los centros a los que asistían los estudiantes que participaron en la investigación. La recogida de información fue realizada mediante la técnica de cuestionario. Estos fueron aplicados, individualmente, sin límite de tiempo y en un único momento temporal por personal especializado que colaboró en el estudio. A los participantes se les recalcó la importancia de responder con sinceridad a todas las cuestiones planteadas. Asimismo, se les informó del carácter totalmente confidencial de la información facilitada.

\section{Técnicas de análisis de datos}

Se procedió al establecimiento de grupos de estudiantes en función de su nivel de atención emocional y claridad emocional. La normalidad de la distribución de los datos, estimada mediante el IQR/S, así como la relación lineal entre las variables, nos sugiere la procedencia de estimar los valores medios de la muestra en las variables atención emocional y claridad emocional mediante el uso de las puntuaciones correspondientes a los percentiles 25,50 y 75 .

Con el objetivo de averiguar si las diferencias observadas en la percepción de estresores y en las respuestas psicofisiológicas de estrés entre los grupos establecidos (baja, media y alta atención emocional y claridad emocional) eran estadísticamente significativas, se realizaron análisis de la varianza (ANOVA). Para averiguar la existencia de diferencias significativas entre los diferentes grupos establecidos, se utilizó Scheffé o Games-Howell como prueba de contraste post hoc, según la posibilidad de asumir o no homogeneidad en las varianzas. En todos los contrastes efectuados se asumió un nivel de significación de 0,05 . 


\section{Resultados}

Atención emocional y percepción de estresores académicos

En la tabla 1 se muestran las puntuaciones medias y desviaciones típicas obtenidas en la escala de estresores por cada uno de los grupos de estudiantes con alta, media y baja atención emocional, así como los valores de la prueba de ANOVA y de los contrastes post hoc. Tal y como puede comprobarse en ella, existen diferencias estadísticamente significativas entre los estudiantes que informan de distintos niveles de atención emocional únicamente para la percepción de estresores que se relacionan directamente con 2 tipos de creencias: por una parte, aquellas que atañen a la carencia de valor de los contenidos y, por otra, las que implican dudas e inseguridad respecto al propio rendimiento académico. Los contrastes a posteriori permiten compro- bar que las diferencias de medias resultan estadísticamente significativas solamente cuando se comparan los grupos de baja y alta atención emocional en los 2 estresores señalados (creencias de rendimiento y carencia de valor) ( $\mathrm{p}=0,028 \mathrm{y} \mathrm{p}=0,009$, respectivamente). Como era previsible, el resto de contrastes no son significativos.

Tabla 1. Medias, desviaciones típicas y significación estadística de las diferencias entre estudiantes con niveles altos, medios y bajos de atención emocional respecto a la percepción de estresores académicos

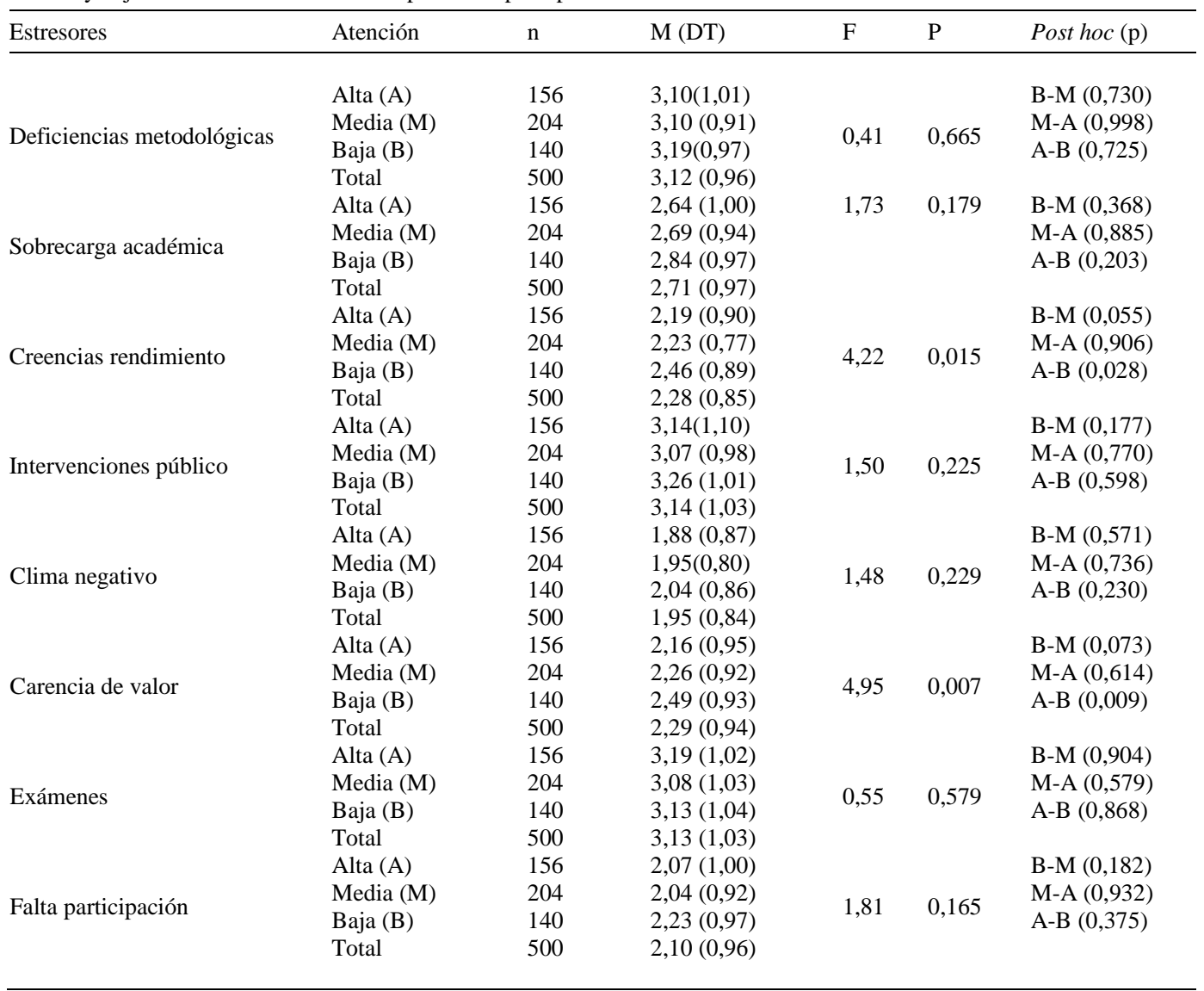




\section{Atención emocional y respuestas psicofisiológicas de estrés}

En la tabla 2 se muestran las puntuaciones medias y desviaciones típicas obtenidas en la escala de respuesta por cada uno de los grupos de estudiantes con alta, media y baja atención emocional, así como los valores de la prueba de ANOVA y de los contrastes a posteriori. En este caso, únicamente se han encontrado diferencias estadísticamente significativas entre los grupos de estudiantes según sus niveles de atención emocional para la dimensión de pensamientos negativos. Aparecen valores medios muy parejos entre los diferentes grupos de atención emocional, mostrando únicamente una puntuación ligeramente superior para los estudiantes que informan de bajos niveles de atención emocional. En este sentido, el único contraste post hoc que casi alcanza significación estadística $(\mathrm{p}=0,060)$ es la comparación baja vs. media atención emocional para el factor pensamientos negativos.

Tabla 2 Medias, desviaciones típicas y significación estadística de las diferencias entre estudiantes con niveles altos, medios y bajos de atención emocional respecto a las respuestas psicofisiológicas de estrés

\begin{tabular}{|c|c|c|c|c|c|c|}
\hline Respuestas psicofisiológicas & Atención & $\mathrm{n}$ & M (DT) & $\mathrm{F}$ & $\mathrm{p}$ & Post hoc $(\mathrm{p})$ \\
\hline \multirow{5}{*}{ Irascibilidad } & Alta (A) & 156 & $1,80(0,92)$ & \multirow{4}{*}{2,91} & \multirow{4}{*}{0,055} & B-M $(0,065)$ \\
\hline & Media (M) & 204 & $1,76(0,84)$ & & & M-A $(0,893)$ \\
\hline & Baja (B) & 140 & $1,99(1,01)$ & & & A-B $(0,206)$ \\
\hline & Total & 500 & $1,84(0,92)$ & & & \\
\hline & Alta (A) & 156 & $1,74(0,84)$ & \multirow{4}{*}{1,21} & \multirow{3}{*}{0,299} & B-M $(0,565)$ \\
\hline \multirow{3}{*}{ Alteraciones del sueño } & Media (M) & 204 & $1,79(0,81)$ & & & M-A $(0,815)$ \\
\hline & Baja (B) & 140 & $1,89(0,94)$ & & & A-B $(0,305)$ \\
\hline & Total & 500 & $1,80(0,86)$ & & & \\
\hline \multirow{4}{*}{ Agotamiento físico } & Alta (A) & 156 & $2,60(1,03)$ & \multirow{4}{*}{1,67} & \multirow{4}{*}{0,190} & B-M $(0,291)$ \\
\hline & Media (M) & 204 & $2,62(0,98)$ & & & M-A $(0,991)$ \\
\hline & Baja (B) & 140 & $2,79(1,1)$ & & & A-B $(0,259)$ \\
\hline & Total & 500 & $2,66(1,0)$ & & & \\
\hline \multirow{4}{*}{ Pensamientos negativos } & Alta (A) & 156 & $1,82(0,94)$ & \multirow{4}{*}{3,20} & \multirow{4}{*}{0,042} & B-M $(0,060)$ \\
\hline & Media (M) & 204 & $1,80(0,80)$ & & & M-A $(0,990)$ \\
\hline & Baja (B) & 140 & $2,04(1,02)$ & & & A-B $(0,130)$ \\
\hline & Total & 500 & $1,87(0,91)$ & & & \\
\hline
\end{tabular}

En suma, y en relación con la primera de las hipótesis, puede afirmarse que los estudiantes de fisioterapia que informan de bajos niveles de atención emocional perciben la creencia sobre el rendimiento y la carencia de valor de los contenidos como más estresantes que los estudiantes con altos niveles de atención emocional. En el resto de los estresores no se encuentra ninguna relación significativa. Tampoco se encuentra relación significativa alguna entre mayores niveles de atención emocional y síntomas psicofisiológicos de estrés. 


\section{Claridad emocional y percepción de estresores académicos}

Por otra parte, en la tabla 3 se recogen las puntuaciones medias y desviaciones típicas obtenidas en la escala de estresores por cada uno de los grupos de estudiantes con alta, media y baja claridad emocional, así como los valores de la prueba de ANOVA y de los contrastes a posteriori. En este caso, existen diferencias significativas en todos los estresores académicos entre los grupos de estudiantes establecidos en función del grado de claridad emocional que poseen, excepto para los estresores vinculados con las deficiencias metodológicas y la falta de participación.

Tabla 3. Medias, desviaciones típicas y significación estadística de las diferencias entre estudiantes con niveles altos, medios y bajos de claridad emocional respecto a la percepción de estresores académicos

\begin{tabular}{|c|c|c|c|c|c|c|}
\hline Estresores & Claridad & $\mathrm{n}$ & $\mathrm{M}(\mathrm{DT})$ & $\mathrm{F}$ & $\mathrm{P}$ & Post hoc (p) \\
\hline \multirow{5}{*}{ Deficiencias metodológicas } & Alta (A) & 83 & $2,96(1,04)$ & \multirow{4}{*}{24,38} & \multirow{4}{*}{0,127} & B-M $(0,532)$ \\
\hline & Media (M) & 334 & $3,13(0,95)$ & & & M-A $(0,357)$ \\
\hline & Baja (B) & 83 & $3,26(0,91)$ & & & A-B $(0,130)$ \\
\hline & Total & 500 & $3,12(0,96)$ & & & \\
\hline & Alta (A) & 83 & $2,48(1,05)$ & \multirow{3}{*}{6,71} & \multirow{3}{*}{0,001} & B-M $(0,024)$ \\
\hline \multirow{3}{*}{ Sobrecarga académica } & Media (M) & 334 & $2,70(0,92)$ & & & M-A $(0,214)$ \\
\hline & Baja (B) & 83 & $3,02(1,01)$ & & & A-B $(0,003)$ \\
\hline & Total & 500 & $2,71(0,97)$ & \multirow{5}{*}{18,92} & & \\
\hline \multirow{4}{*}{ Creencias rendimiento } & Alta (A) & 83 & $1,92(0,81)$ & & \multirow{4}{*}{$<0,001$} & $\mathrm{~B}-\mathrm{M} \ll 0,001)$ \\
\hline & Media (M) & 334 & $2,28(0,79)$ & & & M-A $(0,002)$ \\
\hline & Baja (B) & 83 & $2,69(0,96)$ & & & $A-B \ll 0,001)$ \\
\hline & Total & 500 & $2,28(0,85)$ & & & \\
\hline \multirow{4}{*}{ Intervenciones público } & Alta (A) & 83 & $2,94(1,14)$ & \multirow{4}{*}{3,84} & \multirow{4}{*}{0,022} & B-M $(0,156)$ \\
\hline & Media (M) & 334 & $3,14(0,99)$ & & & M-A $(0,297)$ \\
\hline & Baja (B) & 83 & $3,38(1,04)$ & & & A-B $(0,023)$ \\
\hline & Total & 500 & $3,14(1,03)$ & & & \\
\hline \multirow{4}{*}{ Clima negativo } & Alta (A) & 83 & $1,71(0,80)$ & \multirow{4}{*}{10,49} & \multirow{4}{*}{$<0,001$} & B-M $(0,009)$ \\
\hline & Media (M) & 334 & $1,93(0,79)$ & & & M-A $(0,054)$ \\
\hline & Baja (B) & 83 & $2,28(0,98)$ & & & $A-B \ll 0,001)$ \\
\hline & Total & 500 & $1,95(0,84)$ & & & \\
\hline \multirow{4}{*}{ Carencia de valor } & Alta (A) & 83 & $2,07(0,98)$ & \multirow{4}{*}{3,98} & \multirow{4}{*}{0,019} & B-M $(0,309)$ \\
\hline & Media (M) & 334 & $2,30(0,93)$ & & & M-A $(0,134)$ \\
\hline & Baja (B) & 83 & $2,48(0,90)$ & & & A-B $(0,020)$ \\
\hline & Total & 500 & $2,29(0,94)$ & & & \\
\hline \multirow{3}{*}{ Exámenes } & Alta (A) & 83 & $2,87(1,10)$ & \multirow{4}{*}{3,70} & \multirow{3}{*}{0,026} & B-M $(0,719)$ \\
\hline & Media (M) & 334 & $3,16(1,00)$ & & & M-A $(0,060)$ \\
\hline & Baja (B) & 83 & $3,26(1,03)$ & & & A-B $(0,043)$ \\
\hline \multirow{5}{*}{ Falta participación } & Total & 500 & $3,13(1,03)$ & & & \\
\hline & Alta (A) & 83 & $2,10(1,04)$ & \multirow{4}{*}{1,60} & \multirow{4}{*}{0,202} & B-M $(0,202)$ \\
\hline & Media (M) & 334 & $2,06(0,93)$ & & & M-A $(0,950)$ \\
\hline & Baja (B) & 83 & $2,27(1,00)$ & & & A-B $(0,507)$ \\
\hline & Total & 500 & $2,10(0,96)$ & & & \\
\hline
\end{tabular}

Con relación a las pruebas de contraste a posteriori, las diferencias entre los grupos de alta vs. baja claridad emocional resultan estadística mente significativas para los estresores académicos que también lo eran en la prueba ANOVA, es decir, todos ellos, con las excepciones de deficiencias metodológicas y falta de participación. Especial relevancia tienen los resultados de la dimensión creencias de rendimiento, puesto que es la única en la que las comparaciones intergrupos resultan igualmente significativas cuando se comparan los grupos de nivel alto vs. medio y medio vs. bajo de claridad emocional ( $\mathrm{p}<0,001$, para niveles alto vs. bajo; $\mathrm{p}<0,001$, para niveles bajo vs. medio; $\mathrm{p}=0,002$, para niveles alto vs. medio). Las comparaciones medio vs. 
bajo son estadísticamente significativas además para los factores sobrecarga académica $(\mathrm{p}=0,024)$ y clima negativo $(\mathrm{p}=0,009)$.

\section{Claridad emocional y respuestas psicofisiológicas de estrés}

En la tabla 4 se muestran las puntuaciones medias y desviaciones típicas obtenidas en la escala de respuesta por cada uno de los grupos de estudiantes con alta, media y baja claridad emocional, así como los valores de la prueba de ANOVA y contrastes post hoc. En este caso existen diferencias estadísticamente significativas en la experimentación de las 4 respuestas psicofisiológicas de estrés en función del grado de claridad emocional informado por los estudiantes. En cuanto a los contrastes a posteriori, se encuentran diferencias significativas en los valores medios de las 4 respuestas de estrés consideradas y para todas las comparaciones realizadas entre los 3 grupos de claridad emocional definidos.

Tabla 4 Medias, desviaciones típicas y significación estadística de las diferencias entre estudiantes con niveles altos, medios y bajos de claridad emocional respecto a las respuestas psicofisiológicas de estrés

\begin{tabular}{|c|c|c|c|c|c|c|}
\hline $\begin{array}{l}\text { Respuestas } \\
\text { psicofisiológicas }\end{array}$ & Claridad & $\mathrm{n}$ & M (DT) & $\mathrm{F}$ & $\mathrm{P}$ & Post hoc (p) \\
\hline \multirow{4}{*}{ Irascibilidad } & Alta (A) & 83 & $1,46(0,73)$ & \multirow{5}{*}{22,20} & \multirow{5}{*}{$<0,001$} & B-M $(<0,001)$ \\
\hline & Media (M) & 334 & $1,80(0,83)$ & & & $\mathrm{M}-\mathrm{A}(<0,001)$ \\
\hline & Baja (B) & 83 & $2,35(1,17)$ & & & A-B $(<0,001)$ \\
\hline & Total & 500 & $1,84(0,92)$ & & & \\
\hline \multirow{4}{*}{ Alteraciones del sueño } & Alta (A) & 83 & $1,51(0,74)$ & & & B-M $(<0,001)$ \\
\hline & Media (M) & 334 & $1,76(0,77)$ & \multirow{3}{*}{19,19} & \multirow{3}{*}{$<0,001$} & M-A $(0,019)$ \\
\hline & Baja (B) & 83 & $2,27(1,09)$ & & & A-B $(<0,001)$ \\
\hline & Total & 500 & $1,80(0,86)$ & & & \\
\hline \multirow{4}{*}{ Agotamiento físico } & Alta (A) & 83 & $2,26(1,00)$ & \multirow{4}{*}{14,34} & \multirow{4}{*}{$<0,001$} & B-M $(0,002)$ \\
\hline & Media (M) & 334 & $2,65(1,00)$ & & & M-A $(0,006)$ \\
\hline & Baja (B) & 83 & $3,09(1,05)$ & & & $\mathrm{A}-\mathrm{B}(<0,001)$ \\
\hline & Total & 500 & $2,66(1,03)$ & & & \\
\hline \multirow{4}{*}{ Pensamientos negativos } & Alta (A) & 83 & $1,55(0,87)$ & \multirow{4}{*}{24,66} & \multirow{4}{*}{$<0,001$} & B-M $(<0,001)$ \\
\hline & Media (M) & 334 & $1,81(0,82)$ & & & M-A $(0,039)$ \\
\hline & Baja (B) & 83 & $2,45(1,07)$ & & & A-B $(<0,001)$ \\
\hline & Total & 500 & $1,87(0,91)$ & & & \\
\hline
\end{tabular}

En suma, y en relación con la segunda de las hipótesis, puede afirmarse que cuanto más elevados son los niveles de claridad emocional informados, se perciben como menos estresantes todas las situaciones académicas medidas, con la excepción de las deficiencias metodológicas del profesorado y la falta de participación. También puede afirmarse que cuanto más elevados son los niveles de claridad emocional informados, menores son los niveles de las respuestas psicofisiológicas de estrés medidas. 


\section{Discusión y conclusiones}

En la capacidad para afrontar eficazmente el estrés cada día se evidencia más claramente el papel que desempeñan los recursos intrapsíquicos de la persona, así como su potencial efecto protector. Dentro de ellos, el estudio de las emociones y de su manejo adaptativo adquiere cada vez una mayor relevancia ${ }^{22}$.

En esta investigación se ha abordado el componente experiencial de las emociones, el más básico y directo. En concreto, se analizan las relaciones que mantienen con el estrés académico (con la percepción de estresores académicos y con las respuestas psicofisiológicas) la atención emocional y la claridad emocional.

Con relación al rol desempeñado por la atención emocional, nuestros resultados muestran que, en general, la relación que mantienen los distintos niveles de esta dimensión con el estrés académico es significativa solo en algunas de las variables estudiadas. De hecho, solo encontramos una relación significativa entre baja y alta atención emocional en el caso de los estresores creencias sobre el rendimiento y carencia de valor de los contenidos académicos. Es decir, con los estresores académicos directamente relacionados con creencias del estudiante. Son los estudiantes con menores niveles de atención emocional los que informan de mayo- res niveles de estrés percibido. En este caso los resultados son parcialmente consistentes con los obtenidos por Stratton et al. ${ }^{11}$, que relacionan una disminución en el nivel de atención a las emociones con un incremento en el estrés percibido por estudiantes de medicina. Sin embargo, dada la ausencia de diferencias estadísticamente significativas en la mayoría de los estresores académicos considerados, la relación entre atención emocional y estrés percibido no parece especialmente significativa.

En la misma línea, tampoco hemos encontrado relaciones significativas entre los distintos niveles de atención emocional y las manifestaciones psicofisiológicas de estrés en los estudiantes. Esto concuerda con lo señalado por Costa et al..$^{23}$, quienes no encuentran una relación significativa entre la dimensión de atención y la salud mental de estudiantes universitarios de diferentes países.

La dimensión de claridad emocional se relaciona de forma significativa con la percepción de todos los estresores académicos medidos, con la excepción de la falta de participación y las deficiencias metodológicas del profesorado, estresores más ligados a la organización institucional y en los que el papel de los recursos del estudiante resulta indudable- mente menos significativo. Parece relevante señalar que la relación de esta dimensión resulta mayor con los estresores académicos relacionados con la autoestima, las relaciones sociales y la sobrecarga. Se han puesto de manifiesto evidentes diferencias en la percepción de estresores académicos entre los estudiantes en función de los niveles de claridad emocional mostrados: mientras que los estudiantes con alta claridad emocional presentan bajos niveles de percepción de estresores académicos, los estudiantes con baja claridad emocional valoran las condiciones del entorno académico como más estresantes.

Estos resultados resultan parcialmente coincidentes con la investigación de Extremera et al. ${ }^{12}$, en la que se señala la importante función desempeñada por la claridad emocional sobre las consecuencias del estrés y el bienestar de los estudiantes universitarios. Sin embargo, esta relación se establecería fundamentalmente bajo condiciones interpretadas como altamente demandantes, disminuyendo ante situaciones menos exigentes. En este sentido, parece factible que los estudiantes interpreten las deficiencias metodológicas y las dificultades de participación como situaciones poco modificables y, consecuentemente, menos demandantes que otras en las que su conducta y sus creencias de control juegan un papel más determinante.

Los resultados obtenidos en nuestra investigación ponen de relieve también una relación significativa entre los distintos niveles de claridad emocional y los efectos o respuestas psicofisiológicas de estrés. De tal manera que cuanto más elevado es el nivel de la dimensión claridad emocional, menores son las manifestaciones de estrés tales como irascibilidad, alteraciones del sueño, agotamiento físico o pensamientos negativos. Por tanto, es posible afirmar 
que la claridad emocional puede desempeñar un papel protector frente a la aparición de las consecuencias físicas y psicológicas del estrés académico en estudiantes universitarios. Este resultado concuerda con el trabajo de Ruiz-Aranda et al. ${ }^{8}$, que, con una muestra de estudiantes de ciencias de la salud formada parcialmente por estudiantes de fisioterapia, concluyen que aquellos con altas competencias emociona- les evalúan las situaciones como menos estresantes, lo que revierte en un mayor grado de satisfacción vital y bienestar.

Un reciente trabajo ${ }^{24}$ pone de manifiesto la existencia de diferentes perfiles emocionales en estudiantes de educación secundaria. Si bien existirían un grupo con altas competencias y otro con bajas habilidades emocionales, existen patrones mixtos que se caracterizan por una baja atención emocional. En concreto, este grupo mixto se relaciona con una mayor ansiedad social, aunque la diferencia resulte de pequeña magnitud. Este factor podría contribuir a explicar por qué los estudiantes con baja atención emocional son más propensos a valorar las creencias sobre el rendimiento o la carencia de valor de los contenidos en términos más estresantes. Futuros trabajos deberían analizar el papel que desempeñan diferentes perfiles emocionales en el estrés académico percibido por estudiantes universitarios de ciencias de la salud, que, además de afrontar las demandas comunes de la educación universitaria, deben superar las exigencias psicosociales de la inmersión clínica ${ }^{25}$.

De acuerdo con lo afirmado por Ruíz Aranda et al. ${ }^{8}$ y por Brougham et al. ${ }^{26}$, debido a que la etapa de formación universitaria representa un periodo crítico en el desarrollo de importantes recursos de afrontamiento para el resto de la vida, parece fundamental que la Universidad incremente sus esfuerzos por formar a los estudiantes en habilidades de manejo del estrés, dada su consistente correlación con una disminución en el bienestar físico, psicológico y social.

La capacidad de regular y comunicar emociones de forma efectiva contribuye a mantener relaciones sociales y a reducir los niveles de estrés ${ }^{27}$. En este sentido, la habilidad para procesar adecuadamente emociones y cogniciones conduce a una valoración y afrontamiento más adaptativo de los estresores ${ }^{8,26}$.

Diversos autores ${ }^{8,28}$ han señalado la necesidad de incluir la formación en competencias emocionales dentro de los programas de formación en ciencias de la salud, con la finalidad de dotar a los estudiantes de destrezas que necesitarán en su desarrollo profesional, como el trabajo colaborativo o la interacción con los pacientes y sus familias.

Como todo trabajo de investigación, el presente estudio presenta una serie de limitaciones que es necesario explicitar, a fin de que puedan ser soslayadas de cara al desarrollo de futuros proyectos. La primera de ellas es el diseño metodológico del estudio, que, al ser de tipo transversal, únicamente permite establecer correlaciones pero no atribuir causalidad. Futuros estudios deberían apostar por diseños de tipo longitudinal, en los que fuese posible observar cambios en el nivel de estrés académico tras manipular de forma controlada diferentes variables, en este caso las competencias de regulación emocional de los estudiantes, por ejemplo a través de programas específicos de entrenamiento en el manejo de este tipo de recursos. La segunda es relativa a la especificidad de la muestra, lo que impide la extrapolación de resultados. Pero, a su vez, esta limitación puede también ser una de las virtudes del trabajo, ya que este es uno de los pocos estudios de ámbito nacional e internacional que se ha ocupado de estudiar específicamente el estrés académico en estudiantes de fisioterapia, una población hasta el momento muy poco atendida en comparación con otras como los estudiantes de medicina o enfermería. El hecho de utilizar una muestra tan específica permite caracterizar este fenómeno en este tipo de estudiantes, a la vez que condiciona la generalización de los resultados a otras poblaciones. 


\section{Responsabilidades éticas}

Protección de personas y animales. Los autores declaran que para esta investigación no se han realizado experimentos en seres humanos ni en animales.

Confidencialidad de los datos. Los autores declaran que en este artículo no aparecen datos de pacientes.

Derecho a la privacidad y consentimiento informado. Los autores declaran que en este artículo no aparecen datos de pacientes.

\section{Conflicto de intereses}

Los autores declaran no tener ningún conflicto de intereses.

\section{Bibliografia}

1. Dutta AP, Pyles MA, Miederhoff PA. Stress in health professions students: Myth or reality? A review of the existing literature. J Natl Black Nurses Assoc. 2005;16:63-8.

2. Abdulghani HM. Stress and depression among medical students: A cross sectional study at a medical college in Saudi Aarabia. Pak J Med Sci. 2008;24:12-7.

3. Cabanach RG, Fernández·Cervantes R, González 1, Freire C. Estresores académicos percibidos por estudiantes universitarios de ciencias de la salud. Fisioterapia. 2010;32:151-8.

4. Cabanach RG, Souto -Gestal A, Freire C, Ferradás MM. Relaciones entre autoestima y respuesta de estrés en estudiantes universitarios. Eur J Educ Psychol. 2014;7:43-57.

5. Doherty EM, Nugent E. Personality factors and medical training: A review of the literature. Med Educ. $2011 ; 45: 132-40$.

6. Hernández M, Blavo C, Hardigan P, Pérez A, Hage K. Differences in perceived stress, depression, and medical symptoms among medical, nursing, and physician assistant students: A latent class analysis. Ann Behav Sci Med Educ. 2010;16: 35-9.

7. Por J, Barriball 1, Fitzpatrick J, Roberts J. Emotional intelligence: Its relationship to stress, coping, wellbeing and professional performance in nursing students. Nurse Educ Today. $2011 ; 31: 855-60$.

8. Ruiz-Aranda D, Extremera N, Pineda-Galán C. Emotional intelligence, life satisfaction and subjective happiness in female student health professionals: The mediating effect of perceived stress. J Psychiatr Mental Health Nurs. 2014;21 :106-13.

9. Gratz Kl, Roemer L. Multidimensional assessment of emotion regulation and dysregulation: Development, factor structure, and initial validation of the Difficulties in Emotion Regulation Scale. J Psychopathol Behav. 2004;26:41-54.

10. Stratton TD, Elam Cl, Murphy-Spencer AE, Quinlivan S1. Emotional intelligence and clinical skills: Preliminary results from a comprehensive clinical performance examination. Acad Med. 2005;80:S34-7.

11. Stratton TD, Saunders JA, Elam Cl. Changes in medical stu- dents' emotional intelligence: An exploratory study. Teach Learn Med. 2008;20:279-84.

12. Extremera N, Durán A, Rey L. The moderating effect of trait meta-mood and perceived stress on life satisfaction. Pers Indiv Differ.2009;47:116-21.

13. Saklofske DH, Austin EJ, Galloway J, Davidson K. Individual difference correlates of health-related behaviours: Preliminary evidence for links between emotional intelligence and coping. Pers Indiv Differ. 2007;42:491-502.

14. Kulikowska A, Pokorski M. Self-injuries in adolescents: Social competence, emotional intelligence, and stigmatization. J Physiol Pharmacol. 2008;59 Suppl. 6:383-92.

15. Thompson RJ, Dizén M, Berenbaum $\mathrm{H}$. The unique relations between emotional awareness and facets of affective instability. J Res Pers. 2009;43:875-9.

16. Oliván B, Boira S, López-del-Hoyo Y. Estrés y otros factores psicológicos asociados en estudiantes de fisioterapia. Fisioterapia. $2011 ; 33: 19-24$.

17. Walsh JM, Feeney C, Hussey J, Donnellan C. Sources of stress and psychological morbidity among undergraduate physiotherapy students. Physiotherapy. 2010;96:206-12. 
18. Hervás G, Jódar R. Adaptación al castellano de la Escala de Dificultades en la Regulación Emocional. Clínica y Salud. 2008;19:139-56.

19. Cabanach RG, Souto-Gestal A, Fernández-Cervantes R, Freire C. Regulación emocional y burnout académico en estudiantes universitarios de fisioterapia. REINED. 2011 ;9:7-18.

20. Cabanach RG, Valle A, Rodríguez S, Piñeiro 1. Variables explicativas del estrés en estudiantes universitarios: construcción de una escala de medida. Comunicación. V Congreso Internacional de Psicología y Educación: los retos de futuro. Oviedo (España). 2008:23-5.

21. Cabanach RG, Valle A, Rodríguez S, Piñeiro 1. Respuesta de estrés en contextos universitarios: construcción de una escala de medida. Comunicación. V Congreso Internacional de Psicología y Educación: los retos de futuro. Oviedo (España). 2008:23-5.

22. Friedman HS, Cohen Silver R. Foundations of Health Psychology. New York: Oxford University Press; 2007.

23. Costa H, Ripoll P, Sánchez M, Carvalho C. Emotional intelligence and self-efficacy: Effects on psychological well-being in college students. Span J Psychol. 2013;16:E50.

24. Gázquez JJ, Pérez-Fuentes MC, Diaz-Herrero A, García- Fernández JM, Inglés CJ. Perfiles de inteligencia emocional y conducta social en adolescentes españoles. Psicol Conduc. 2015;23:141.

25. Sanjuán A, Ferrer ME. Perfil emocional de los estudiantes en prácticas clínicas: acción tutorial en enfermería para apoyo, formación, desarrollo y control de las emociones. Inv Educ Enferm. 2008;26:22635 .

26. Brougham RR, Zail CM, Mendoza CM, Miller JR. Stress, sex differences, and coping strategies among college students. Curr Psychol. 2009;28:85-97.

27. Skowron EA, Wester SR, Azen R. Differentiation of self mediates college stress and adjustment. J Couns Dev. 2004;82:69-78.

28. Stoller JK, Taylor CA, Farver CF. Emotional intelligence competencies provide a developmental curriculum for medical training. Med Teach. 2013;35:243-7. 